\title{
Die operative Behandlung von Skaphoidfrakturen
}

\author{
Jörg Schmidt, Karl Heinrich Winker
}

\section{Zusammenfassung}

Der Kahnbeinbruch war und ist vielfach auch noch immer eine Domäne der konservativen Behandlung. Ruhigstellungen von 6 bis 16 Wochen, teilweise im Oberarmgipsverband, sind jedoch bis zur Ausheilung notwendig. In neuerer Zeit finden vermehrt operative Verfahren Anwendung, die durch Einführung moderner Implantate und Verfahren eine Ruhigstellung umgehen lassen und bei sehr geringer Komplikationsrate eine verbesserte Heilungsrate zeigen.

\section{Einleitung}

Der Bruch des Kahnbeins ist eine relativ seltene Fraktur. Nur 1 - 4\% aller Knochenbrüche beim Menschen machen eine Fraktur dieses kleinen Knochens des Karpus aus. Sieht man jedoch nur die Handwurzel, so liegt ein Kahnbeinbruch in etwa 35\% aller Frakturen vor [5].

Bis vor wenigen Jahren wurde die Behandlung der Kahnbeinbrüche als ein in der Hauptsache konservatives Problem angesehen, so wie es bereits Böhler beschrieben hat. Er sah eine ununterbrochene Ruhigstellung in einem ungepolsterten Gips für in der Regel 6Wochen indiziert, sollte dann keine Heilung eingetreten sein, war eine Ruhigstellung bis zu 16Wochen angezeigt [1].

OP-JOURNAL 2001; 17: 264-268

(c) Georg Thieme Verlag Stuttgart · New York
Diese Auffassung ist bis in die jüngste Vergangenheit hinein unwidersprochen. Noch 1991 wurde an gleicher Stelle beschrieben, dass die frische Kahnbeinfraktur unbestritten die Domäne der konservativen Therapie sei [6].

1996 berichten Stuttmann und Rudolph [7], dass bei der konservativen Therapie 95 bis über $98 \%$ aller frischen Skaphoidfrakturen bei korrekter Gipsruhigstellung ausheilen würden. Ruhigstellungszeiten werden auch hier zwischen 6 und 12 Wochen, ggf. auch bis zu 16 Wochen berichtet.

Solch lange Gipsruhigstellungen haben natürlich erhebliche Probleme und Mängel. So muss zum Beispiel bei einem wenig gepolsterten, gut anmodellierten Gips immer auf Druckschäden geachtet werden, Beschädigungen des Gipses müssen rechtzeitig erkannt und ausgebessert werden, da Unruhe im Gips mit Sicherheit zu einem Nichtdurchbau der Fraktur führen wird. Kontrollröntgenaufnahmen im Verlauf sind aufgrund der Gipsüberlagerung und der oftmals nur feinen Frakturlinie des Skaphoids kaum zu sehen. Auch sind Frakturen des proximalen Drittels des Skaphoids oftmals im Rahmen einer konservativen Therapie nicht zur Ausheilung zu bringen.

Nicht zu übersehen sind jedoch auch die allgemeinen Risiken der konservativen Behandlung, also die Dystrophiegefahr, die Muskelatrophie, die Bewegungseinschränkung der Gelenke einer oberen Extremität, insbesondere dann, wenn ein Oberarmgips angelegt wird.

Neben der mittlerweile unbestrittenen größeren Sicherheit der operativen Behandlung in Bezug auf den Durchbau, insbesondere bei kritischen proximalen Frakturen, ist der Patientenkomfort, d.h. die nicht mehr notwendige Gipsruhigstellung bei freier Beweglichkeit der oberen Extremität, in den letzten Jahren so zunehmend in den Vordergrund getreten, dass der Anteil der operativ versorgten Kahnbeinbrüche ständig ansteigt. Als weiterer großer Vorteil ist zu werten, dass körperlich nicht belastete Patienten mit einem Kahnbeinbruch viel früher als bei der konservativen Behandlung mit all seinen Problemen wieder dem Arbeitsleben zugeführt werden können [3]. Nicht zuletzt aus diesem Grund stellt die operative Behandlung auch der unkomplizierten Kahnbeinbrüche eine Alternative dar, die einem solchen Verletzten angeboten werden muss.

Die Kahnbeinfraktur ist nicht mehr unwidersprochen die Domäne der konservativen Behandlung!

\section{Diagnostik}

Die Diagnostik einer frischen Kahnbeinfraktur beginnt mit der Verdachtsdiagnose. Beim Sturz auf die ausgestreckte Hand und dem Ausschluss einer Fraktur des körperfernen Speichenendes muss immer an einen Kahnbeinbruch gedacht werden. Bei der klinischen Untersuchung des Handgelenkes fällt im der Regel ein Spontanschmerz im Bereich des Daumenballens auf, der Tabatieredruckschmerz ist eines der sichersten Zeichen für die Verdachtsdiagnose eines Kahnbeinbruches.

Seit Lorenz Böhler [1] hat sich das so genannte Skaphoidquartett, eine Röntgenaufnahme der radialen Handwurzelreihe in 4 Ebenen, bewährt. Dazu werden die Einstellungen dorso-palmar in Mittelstellung des Handgelenkes, eine streng seitliche Aufnahme, eine Überstreckaufnahme der Hand und eine so genannte Schrägaufnahme in halber Supinationsstellung durchgeführt. In letzter Zeit häufiger diskutiert wird auch die a.p. Aufnahme in Ulnaabduktionsstellung, da hier das Skaphoid maximal ausgestreckt beurteilt werden kann, ebenso wieder die bewährte Skaphoidvergrößerungsaufnahme. 
Sollten sich Diskrepanzen zwischen der führenden Klinik mit Verdacht einer Skaphoidfraktur und einem negativen Röntgenbild ergeben, können prinzipiell zwei Wege beschritten werden. Zum einen besteht die Möglichkeit, nach einer 10-tägigen Ruhigstellung des Handgelenkes Kontrollröntgenaufnahmen durchzuführen. Hier ist mit Sicherheit dann bereits ein Resorptionssaum im Bereich des Skaphoids zu sehen und somit lässt sich mit an Sicherheit grenzender Wahrscheinlichkeit ein Kahnbeinbruch, der in den Primäraufnahmen durch seine unverschobene Lage und damit nur sehr feine und mit einem Gefäßkanal zu verwechselnde Aufhellungslinie übersehen wurde, darstellen. Möchte man diese 10 Tage nicht abwarten, haben wir mit dem Computertomogramm in semikoronarer Schichtung, also Schichtungen in der Längsachse des Skaphoids, ein sicheres bildgebendes diagnostisches Verfahren zur Hand. In keinster Weise aussagekräftig ist die Knochenszintigraphie, die einzig und allein eine Anreicherung auch bei Bandverletzungen bietet. Wenig aussagekräftig für diese Diagnose ist ebenfalls das MRT, auch hier können Frakturen entweder übersehen oder durch eine Knochenkontusion, dem so genannten bone bruise, vorgetäuscht sein.

Bei klinischem Verdacht auf einen Kahnbeinbruch muss die Diagnose mit Sicherheit gestellt oder ausgeschlossen werden.

$\mathrm{Zu}$ bevorzugen ist also bei unsicherer Diagnostik die Computertomographie, die heute eigentlich allseits schnell durchführbar ist und mittlerweile auch kostengünstig angefertigt werden kann. Ein weiterer, nicht zu unterschätzender Vorteil der Computertomographie ist die Möglichkeit, die Kahnbeinfraktur sicher zu klassifizieren.

\section{Klassifikation}

Lorenz Böhler hat bereits 1935 die erste Klassifikation des Kahnbeines erstellt, wobei er hier das Augenmerk auf die Biomechanik der Frakturlinie und der damit verbundenen Abrutschgefährdung der Fraktur gelegt hat.

Watson-Jones hat 1976 eine Klassifikation entwickelt, in der er die Durchblutungssituation des Skaphoids zugrunde gelegt hat.

T. Herbert, der Vater der modernen Skaphoidbehandlung, hat eine eigene Klassi-
Tab. 1 AO-Klassifikation Karpalknochen

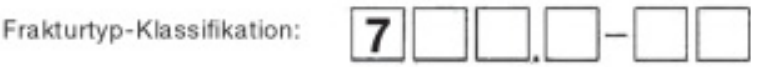

\section{A)}

Abriss, Abscherfrakturen:
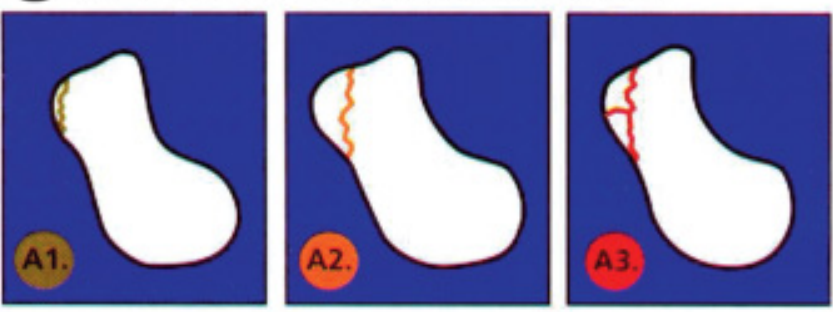

B Frakturen quer, schräg oder parallel zur Unterarm-Längsachse (alle Carpalia)
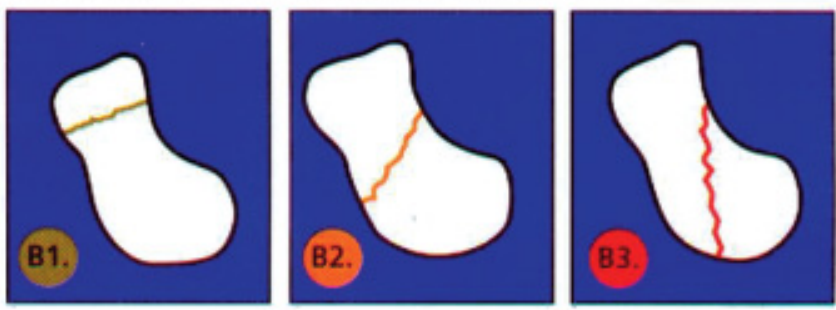

(C)

Mehrfragment- und Trūmmerfrakturen: (alle Carpalia)
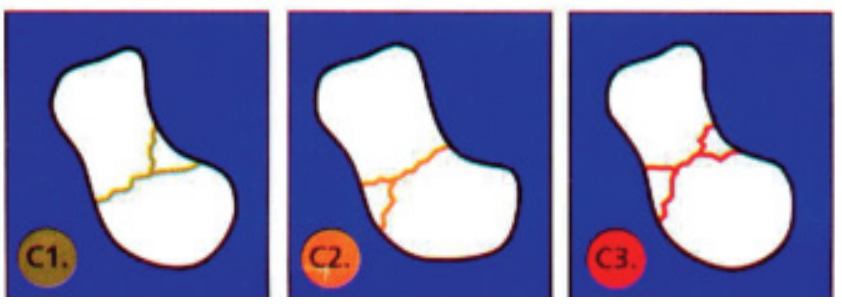

fikation entwickelt [1], in der er zwischen stabilen, instabilen und Polfrakturen unterscheidet und ebenfalls die Skaphoidpseudarthrose in diese Klassifikation mit einbezieht.

Allgemein gebräuchlich, da international anerkannt, sollte die Klassifikation der AO sein (Tab.1).

\section{Implantate}

Zur operativen Versorgung der Skaphoidfraktur wird im AO-Manual noch immer die Kleinfragmentschraube bzw. die Mini-Fragmentschraube bevorzugt. Diese Versorgungen sind ausreichend diskutiert und in ihren Vor- und Nachteilen beleuchtet. Neuentwickelt wurde eine 3,0 $\mathrm{mm}$ kanülierte Schraube mit einer Schraubunterlegscheibe. Diese Schraube zeichnet sich durch eine extrem hohe Zugkraft aus, durch die versenkbare Unterlegscheibe ist dieses Implantat voll im Skaphoid versenkt. Zur Implantation muss jedoch hier das STT-Gelenk in einer relativ großzügigen Art und Weise freigelegt werden.

Das Verdienst von Herbert ist die Entwicklung des modernen Implantates zur Versorgung der Skaphoidfraktur. Er entwickelte eine Doppelgewindeschraube, bei der durch unterschiedliche $\mathrm{Ge}$ windesteigungen eine Kompression innerhalb des Skaphoids aufgebaut werden kann, die Insertion ins Skaphoid beruht auf einem relativ kleinen Eintrittspunkt, ebenfalls innerhalb des STT-Gelenkes. Zur Implantation dieser originären sogenannten Herbert-Schraube muss der Karpus jedoch von palmar oder dorsal eröffnet, das Skaphoid muss dargestellt und aus einem Teil seiner Bandverbindungen gelöst werden. Dies ist dazu notwendig, um das entsprechende Zielinstumentarium in die radiokarpale Gelenkfläche auf den proximalen Pol des Skaphoids zu setzen und gleichzeitig das so genannte Kompressionsgig auf den distalen Ska- 
phoidpol aufzusetzen. Diese Schraube wurde mannigfaltig modifiziert. In der heutigen Zeit bieten verschiedene Anbieter Doppelgewindeschrauben an, zum Teil mit verschiedenen Gewindesteigungen und in verschiedenen Größen, um auch kleine proximale Polfragmente mit einer Schraube versorgen zu können.

Die entscheidende Weiterentwicklung ist jedoch die kanülierte Doppelgewindeschraube, die uns heutzutage die perkutane Versorgung dieser Skaphoidfrakturen erlaubt. Grundlage hierfür ist jedoch auch ein Philosophiewandel, der von der Versorgung der Skaphoidfraktur mit einer reinen Kompressionsosteosynthese dazu übergegangen ist, über einen gut sitzenden intraossären Kraftträger die zur Pseudarthroseentwicklung führenden Kippbewegungen des Skaphoids, die aus seiner mechanischen Schaltstelle zwischen proximaler und distaler Handwurzelreihe resultieren, auszuschalten [8]. Daher legen die modernen Implantate keinen gesteigerten Wert mehr auf maximale Kompressionsleistung, sondern vielmehr auf eine sichere interossäre Verankerung und Stabilisierung.

\section{OP-Methode}

\section{Offenes Einbringen der Schraube}

Da sich der größte Anteil der Skaphoidfrakturen im Bereich des mittleren Drittels abspielen, hier werden Zahlen zwischen $50 \%$ und $67 \%$ berichtet, ist der palmare Zugang zum Skaphoid der häufigste. Nach Längsinzision im Bereich der radialen Handwurzelreihe wird der Flexor carpi radialis dargestellt. Durch den Boden des Sehnenfaches hindurch wird scharf in einem Schnitt der Karpus eröffnet. Nach Einsetzen eines Spreizers ist das Skaphoid in ganzer Länge einsehbar. Nach Durchtrennen der Bandverbindungen von Trapezium und Trapezoideum, ist das Kahnbein in der Regel mit zwei Hohmann-Hebeln soweit aus dem Verbund heraus zu luxieren, dass nach Einsetzen des Kompressionsgigs auf den proximalen Skaphoidpol und Aufbringen der Kompressionshülse auf den distalen Pol unter Bildwandlerkontrolle dieses Skaphoid in der Regel reponiert und komprimiert ist. Mit entsprechenden Bohrern kann nun der Knochen aufgebohrt und die Doppelgewindeschraube eingebracht werden. Nach Reposition des Kahnbeines wird unter Einlegen einer Redondrainage die Gelenkkapsel verschlossen, die Sehne reponiert und die Haut ebenfalls verschlossen.

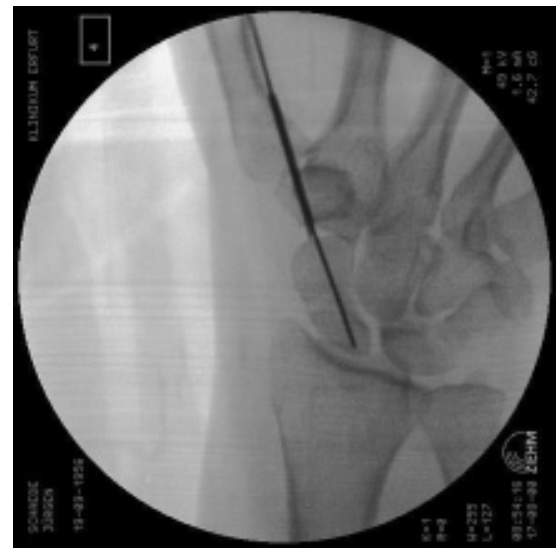

Abb.1 Führungshülse mit Zieldraht.

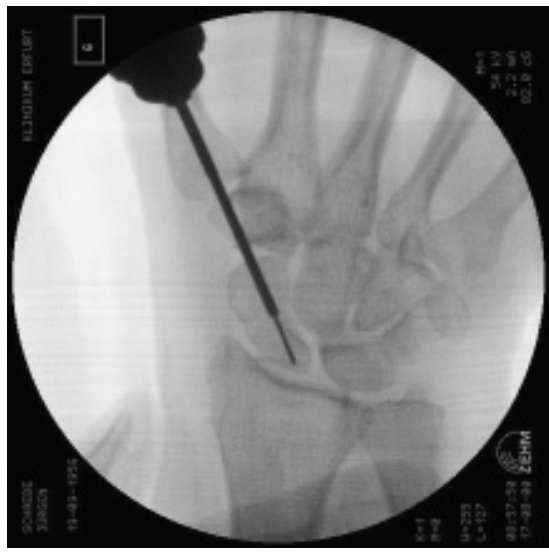

Abb.3 Aufbohren des Skaphoides.

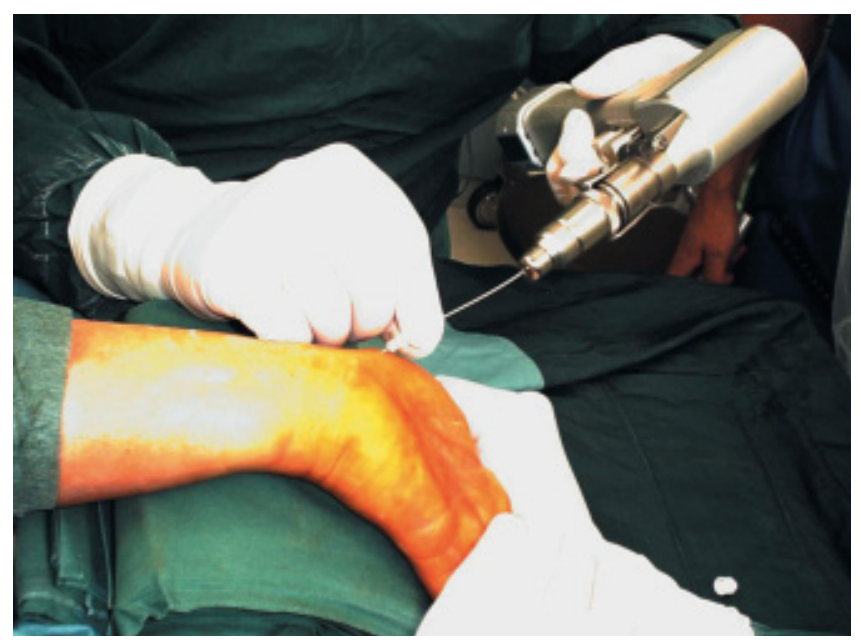

Abb. 2 Einbringen des Zieldrahtes.
Der Vorteil dieser offenen Methode ist, dass dislozierte Skaphoidfrakturen unter Sicht reponiert werden können, auch bei groben Dislokationen ist eine anatomische Reposition der Fraktur unter Aufrichten des interskaphoidalen Winkels möglich. Wenn das Repositionsgig mitbenutzt wird, kann auch hier eine maximale Kompression auf die Fraktur gebracht werden.

Die großzügige Arthrotomie muss auch angewendet werden, wenn selten eingebrachte Implantate, wie z.B. die EnderPlatte, zur Anwendung kommen.

\section{Perkutane Operationsmethode}

Aufgrund der erheblichen Präparationsleistung und der Durchtrennung von Bandverbindungen im Bereich des Kahnbeines haben sich in letzter Zeit minimalinvasive Methoden durchgesetzt. Neben der Methode der Miniarthrotomie, ein kleiner Längsschnitt über dem tastbaren Tuberculum des Skaphoids, haben sich rein perkutane Methoden bewährt. Dazu wird unter Benutzung einer Führungshülse ein Ziel-Kirschner-Draht vom Thenar aus in das Skaphoid eingebracht (Abb.1 u.2). Die Kontrolle der Lage des Kirschner-Drahtes erfolgt unter Bildwandlersicht. Dazu sind natürlich exakte Einstellungen notwendig, um eine Fehllage dieses Implantates zu verhindern. Über diesen Kirschner-Draht wird dann das Skaphoid mit einem standardisierten Bohrer aufgebohrt (Abb.3) und die in der Regel selbstbohrende, selbstschneidende Schraube eingedreht. Unter nochmaliger Kontrolle mit dem Bildwandler ist zu erkennen, wie die Schraube im Kahnbein liegt (Abb.4u.5).

Der Vorteil dieser Operationsmethode liegt zum einen in der äußerst geringen Weichteiltraumatisierung (Abb.6) und in geübter Hand in erheblich kürzeren Operationszeiten. Der Nachteil ist jedoch, dass insbesondere Rotationsfehlstellungen des Kahnbeines in manchen Fällen nicht erkannt und damit nicht reponiert 


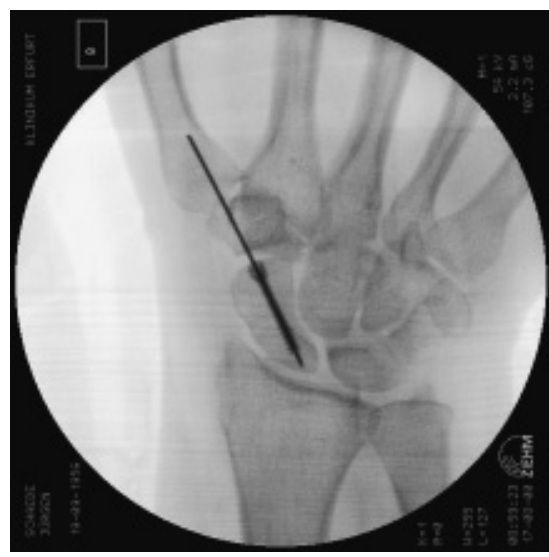

Abb.4 Doppelgewindeschraube mit Zieldraht.

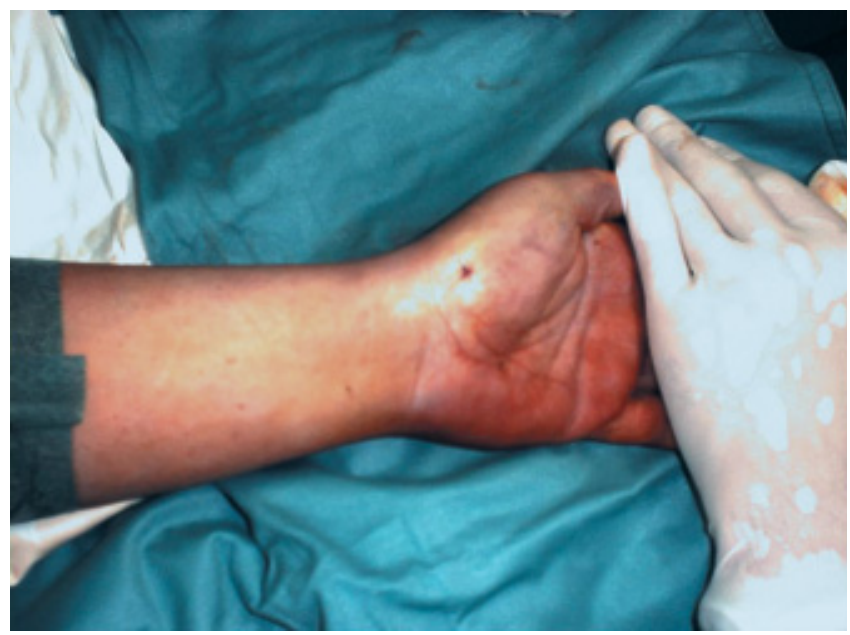

Abb. 6 Hautinzision bei OP-Ende.

werden. Sollte eine solche Rotationsfehlstellung erkannt werden, ist intraoperativ der Umstieg auf das offene Verfahren zwingend notwendig.

Die perkutane Verschraubung des Kahnbeines ist zu bevorzugen, bei Komplikationen muss aber auch die offene Operationstechnik beherrscht werden.

\section{Nachbehandlung}

Bei den operativen Versorgungen des Skaphoids ist eine Gipsruhigstellung selbstverständlich erheblich kürzer notwendig, als sie bei dem konservativen Verfahren angewendet werden muss. In unserer Hand hat es sich bewährt, bei dem offenen Verfahren eine kurzfristige Gipsruhigstellung bis zur sicheren Wundheilung, in der Regel für zwei Wochen, durchzuführen. Bei der perkutan durchgeführten Verschraubung des Skaphoids ist eine Gipsruhigstellung nicht notwendig. Hier erfolgt postoperativ eine elastische Wickelung, der Patient

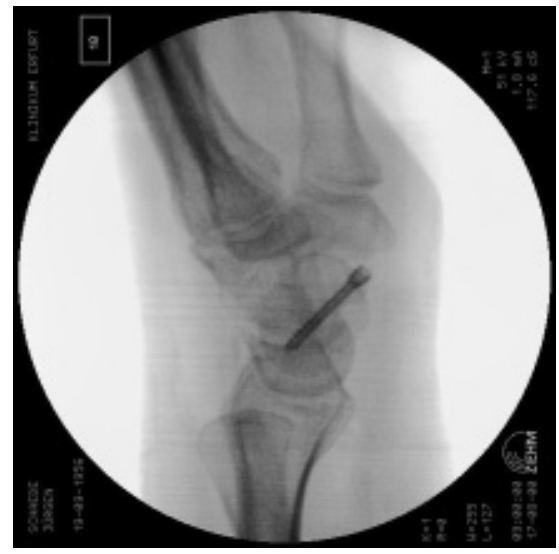

Abb. 5 Schraubenlage seitlich.

darf bis zum Ablauf der 6. Woche das Handgelenk voll bewegen, jedoch nicht belasten. Nach Ablauf der 6. Woche wird eine Röntgenaufnahme und im Zweifelsfall

auch

eine Computertomographie durchgeführt, um den Durchbau des Kahnbeines zu beweisen. In der Regel ist die Knochenheilung nach diesen 6 Wochen so weit vorangeschritten, dass eine zunehmende Belastung des Handgelenkes möglich ist.

Durch die frühzeitige Bewegung des Handgelenkes sind in einem von uns nachuntersuchten Patientenkollektiv keine Bewegungseinschränkungen und insbesondere keine dystrophen Veränderungen des Handgelenkes bzw. der oberen Extremität zu verzeichnen gewesen. Auch die Zufriedenheit der Patienten ist nach diesem operativen Eingriff sehr hoch, insbesondere, da dieses Verfahren äußerst wenige Komplikationen zeigt und wir in fünf Jahren der Anwendung noch keinen einzigen Infekt vorweisen mussten.

Der Vorteil aller operativen Stabilisierungen des Kahnbeines liegt in der frühzeitigen funktionellen Nachbehandlung und des daraus resultierenden Patientenkomforts.

\section{Diskussion}

Die Fraktur des Kahnbeines ist, obwohl sie nur in 1-4\% aller Fälle vorkommt, eine problematische Behandlung. Zum einen wird oftmals von dem gestürzten Patienten die leider nur kurze Schmerzsymptomatik des Handgelenkes unterschätzt, so dass hier Kahnbeinbrüche ohne Behandlung bleiben, zum anderen werden immer wieder Fälle berichtet, in denen Kahnbeinbrüche bei der primären Behandlung übersehen wurden und hier trotz entsprechender Klinik keine weiterführenden diagnostischen Maßnahmen ergriffen werden konnten.

Die konservative Behandlung des Kahnbeinbruches ist mit seiner 6- bis 16-wöchigen Gipsruhigstellung mit einem sehr hohen Dyskomfort für den Patienten behaftet, nicht außer Acht lassen dürfen wir die Probleme der konservativen Behandlung an und für sich, also Ruhigstellungsschäden, Druckschäden im Gips und die oftmals schwierige Führung der in der Regel jungen Patienten. Nicht zu unterschätzen ist in der heutigen Zeit jedoch auch der lange Ausfall aus dem Arbeitsleben, der insbesondere bei Arbeitnehmern aus dem Dienstleistungs- und Informationsgewerbe sowie bei Verwaltungsbeschäftigten nicht mehr sein muss. Aus diesem Grunde sollten allen Patienten operative Maßnahmen vorgeschlagen und diskutiert werden. Der Patientenkomfort steht in der letzten Zeit deutlich im Vordergrund.

Das Kahnbein hat aufgrund seiner zentralen Stellung im Karpus eine immense Bedeutung für die Kinematik des Handgelenkes. Eine hier eingetretene Pseudarthrose, sei es aufgrund unzureichender Behandlung, sei es aufgrund nicht erkannter Frakturen, hat durch den sogenannten karpalen Kollaps (SNAC-Wrist) eine erhebliche Bedeutung für die Arthrosebildung im Bereich des Handgelenkes, spät erkannte Pseudarthrosen machen in der Regel Zweit- und Dritteingriffe notwendig, die oftmals mit erheblichen Bewegungseinschränkungen und verbliebener Beschwerdesymptomatik des Handgelenkes einher gehen [4]. 
Eine übersehene oder nicht behandelte Skaphoidfraktur kann zur Katastrophe für das Handgelenk werden!

Verstümmelnde Eingriffe, wie z.B. die Entfernung des Kahnbeines, Entfernung der proximalen Handwurzelreihe oder gar die Handgelenkarthrodese, stehen am Ende eines solchen Leidensweges. Aus diesem Grunde ist neben einer konsequenten Diagnostik der frischen Kahnbeinfraktur ihre operative Behandlung heutzutage zeitgemäß.

\section{Literatur}

1. Böhler, L. Die Technik der Knochenbruchbehandlung in Frieden und im Kriege. Verlag Wilhelm Maudrich, Wien 1943

2 Eisenschenk A, Lautenbach M, Weber U. Scaphoidfrakturen und Scaphoidpseudarthrosen. Orthopäde 1999; 10: 883-890

3 Herbert TJ. The Fractured Scaphoid. Quality Medical Publishing, St. Louis, Missouri 1990

4 Martini AK. Der Spontanverlauf der Kahnbeinpseudarthrose. Orthopäde 1994; 23 : 249-254

5 Roesgen M, Hierholzer G. Die klinische Problematik der Kahnbeinfraktur. Unfallchirurg 1988; 14: 139-147

6 Roesgen M, Hierholzer G. Osteosynthesen im Bereich der Handwurzel. OP-Journal 1991; 2: $50-54$

7 Studtmann V, Rudolph H. Die Behandlung der Scaphoidfraktur. In: Rahmanzadeh R, Meißner A, Würtenberger C, Fell M. Unfallund Wiederherstellungschirurgie des Unterarmes. Einhorn-Presse Verlag, Reinbek 1996

8 Toby EB, Butler TE et al. A Comparison of Fixation Srews for the Scaphoid during Application of Caclical Bending Loads. JBJS 1997; 79-A: $1190-1197$
Dr. med. Jörg Schmidt

Ltd. Oberarzt

Prof. Dr. med. Karl Heinrich Winker

Chefarzt

Klinik für Unfallchirurgie, Hand- und Wiederherstellungschirurgie am HELIOS-Klinikum Erfurt

Nordhäuser Str. 74

D-99089 Erfurt 